\title{
Circumstellar interaction in Type IIn supernovae
}

\author{
Poonam Chandra ${ }^{1}$, Roger A. Chevalier ${ }^{2}$, Nikolai Chugai ${ }^{3}$, \\ Alicia M. Soderberg ${ }^{4}$, and Claes Fransson ${ }^{5}$ \\ ${ }^{1}$ National Centre for Radio Astrophysics, Pune University Campus, Ganeshkhind, \\ Pune-411 007, INDIA \\ email: poonam@ncra.tifr.res.in \\ ${ }^{2}$ Dept. of Astronomy, University of Virginia, P.O. Box 400325, Charlottesville, VA 22904, USA \\ ${ }^{3}$ Institute of Astronomy of Russian Academy of Sciences, Pyatnitskaya Street 48, 109017 \\ Moscow, Russia \\ ${ }^{4}$ Smithsonian Astrophysical Observatory, 60 Garden Street, Cambridge, MA 02138, USA \\ ${ }^{5}$ Department of Astronomy, Stockholm University, AlbaNova, SE-106 91 Stockholm, Sweden
}

\begin{abstract}
Type IIn supernovae have bright optical emission and high bolometric luminosities. Due to their high mass loss, their are expected to have dense circumstellar interaction, thus produce bright radio and X-ray emission. We aim to carry out systematic study to understand their circumstellar interaction, mass loss properties. Here, I provide specific examples of two Type IIn supernovae, 2006jd and 2010jl.
\end{abstract}

Keywords. dense matter, radiation mechanisms: general, circumstellar matter, stars: mass loss, supernovae: individual (2006jd, 2010jl)

\section{Introduction}

Type IIn supernovae (hereafter SNe IIn) usually have bright optical emission and high bolometric luminosities. They show characteristic narrow emission lines of hydrogen and helium (Schlegel 1990, Filippenko 1997), which are indicative of dense circumstellar medium (CSM). This class of supernovae (SNe) generally show significant heterogeneity in terms of their emission line profiles, luminosity and mass loss rates. In SNe IIn radio, $\mathrm{X}$-ray and late time optical emission comes from circumstellar interaction of the ejecta with the CSM. When the ejecta plow into the CSM with supersonic speeds, a forward shock moves into the circumstellar wind generating $\sim 100 v_{4}^{2} \mathrm{keV}$ plasma, and a reverse shock in the freely expanding SN ejecta generating $\sim 1 v_{4}^{2} \mathrm{keV}$ plasma, where $v_{4}$ is the forward shock velocity in units of $10^{4} \mathrm{~km} \mathrm{~s}^{-1}$. Radio emission is expected to be nonthermal synchrotron emission from the forward shock, while X-ray emission is likely to have a thermal origin arising in both reverse and forward shock Chevalier 1982, Chevalier \& Fransson 2003. Because of the high density of the CSM, the emission due to the CSM interaction is expected to be high. SNe IIn are relatively X-ray luminous, exceeding $10^{40} \mathrm{erg} \mathrm{s}^{-1}$. However, they have remain elusive at radio wavelengths. Out of couple of hundred total SNe IIn, 81 have been looked in radio bands with only 10 detected. This is merely $5 \%$ of total SNe IIn and $12 \%$ of observed.

To understand the progenitor, evolution and mass loss history of SNe IIn, we are carrying out a campaign to study them in multiwavebands with first observations initiated in radio bands. The detected ones are followed up with Chandra, XMM-Newton or Swift-XRT in X-ray bands. So far we have observed a total of $43 \mathrm{SNe}$ IIn with the Very 
Large Array (VLA) †. We have been able to detect radio emission from 4 of them, which are SN 2005kd, 2006jd, 2008iy and 2009ip. In X-ray we have detected SN 2006jd (with Chandra and XMM-Newton), 2010jl (with Chandra at 3 epochs) and SN 2009ip (with $X M M$-Newton). Here we describe SN 2006jd and 2010jl.

SN 2006jd was discovered on 2006 October 12, with an apparent magnitude of 17.2, in the galaxy UGC 4179 at a redshift of $z=0.0186$ (Prasad \& Li 2006). The initial spectrum of SN 2006jd showed features of a Type IIb SN, similar to SN 1993J (Blondin et al. 2006). However, based on Keck spectra at late epochs, it was reclassified as a Type IIn SN (A. Filippenko, 2006, private communication). On the basis of a spectrum on 2006 October 17.51 UT, we assume its explosion date to be 2006 October $06.5 \mathrm{UT}$ (Blondin et al. 2006). Immler et al. (2007) detected X-ray emission from SN 2006jd on 2007 November 16 with the Swift X-ray Telescope (XRT) in a 2.3 ks exposure. The net count rate was $(6.3 \pm 2.0) \times 10^{-3}$ counts $\mathrm{s}^{-1}$. The first radio emission was detected from the SN on 2007 November 21.28 UT in the $5 \mathrm{GHz}$ band, on 2007 November 26.36 UT in the $8.5 \mathrm{GHz}$ band, and on 2007 November $26.38 \mathrm{UT}$ in the $22.5 \mathrm{GHz}$ band Chandra \& Soderberg 2007.

SN 2010jl was discovered on 2010 November 3.5 (UT) at a magnitude of 13.5 (Newton \& Puckett 2010), and brightened to magnitude 12.9 over the next day. Pre-discovery observations indicate an explosion date in early 2010 October (Stoll et al. 2011). Spectra on 2010 November 5 showed it to be a Type IIn event (Benetti et al. 2010). The apparent magnitude is brightest for a Type IIn SN since SN 1998S. SN 2010jl is associated with the galaxy UGC 5189A at a distance of $50 \mathrm{Mpc}(z=0.011)$, implying that SN 2010jl reached $M_{V} \sim-20$ (Stoll et al. 2011) and placing it among the more luminous Type IIn events (Kiewe et al. 2012). Hubble Space Telescope (HST) images of the site of the SN taken a decade before the SN indicate that the progenitor star had an initial mass $\geqslant 30 M_{\odot}$ (Smith et al. 2011). The Swift on board X-ray Telescope (XRT) detected X-rays from SN 2010jl on 2010 November 5.0-5.8 (Immler et al. 2010).

\section{Observations}

\subsection{SN 2006jd Observations}

SN 2006jd was observed in radio bands with the VLA from 2007 Nov 21 onwards and until 2012 Apr 7 in the $22.5 \mathrm{GHz}$ (K band), 8.5 GHz (X), $5 \mathrm{GHz}(\mathrm{C})$, and $1.4 \mathrm{GHz}(\mathrm{L})$ bands at various epochs, along with a $44 \mathrm{GHz}$ band observation at one epoch. For $\mathrm{C}$ and $\mathrm{X}$ bands, the data were taken in the interferometric mode for an average of 30 minutes (including calibrator time), whereas for $\mathrm{L}$ and $\mathrm{K}$ bands the data were collected for $1 \mathrm{hr}$. The data was analysed using standard AIPS routines. We also observed SN 2006jd with the Giant Metrewave Radio Telescope (GMRT) on three occasions between 2009 October to 2010 April in the $1.3 \mathrm{GHz}$ and $0.61 \mathrm{GHz}$ bands and detected in the $1.3 \mathrm{GHz}$ band at both occasions.

SN 2006jd was observed with the Swift on board XRT at 18 epochs between 2007 November and 2011 March in Photon counting mode. All the Swift observations were for exposures less than $10 \mathrm{ks}$. We also observed SN 2006jd once with the Chandra and once with the $X M M$-Newton X-ray observatories. We carried out the XMM-Newton observations starting 2009 April 7 at 4:36:46 UT with the EPIC-PN and EPIC-MOS cameras in full frame with thin filter mode. The exposures for the EPIC-MOS1 and EPIC-MOS2 were $42.667 \mathrm{ks}$ and $42.672 \mathrm{ks}$, respectively, and for the EPIC-PN, the exposure time

$\dagger$ The National Radio Astronomy Observatory is a facility of the National Science Foundation operated under cooperative agreement by Associated Universities, Inc. 
was $41.032 \mathrm{ks}$. We used the EPIC-PN observations to carry out a detailed spectroscopic analysis and 1963 counts were obtained in the $0.2-10 \mathrm{keV}$ range Chandra et al. 2012b. We also observed SN 2006jd with Chandra ACIS-S in VFAINT mode. The observations started on 2009 September 14 at 00:03:21 UT for a total exposure of $37.24 \mathrm{ks}$. A total of 888 counts were obtained in the $0.2-10 \mathrm{keV}$ range Chandra et al. $2012 \mathrm{~b}$.

\subsection{SN 2010jl Observations}

We started radio observations of the SN 2010jl with the VLA starting 2010 Nov 06. The last observation was taken on 2013 Jan 19. The observations have been made in K, C and $\mathrm{X}$ bands. So far we have not detected the $\mathrm{SN}$ in radio bands. We are continuing to follow it in radio bands.

The Swift detection of SN 2010jl allowed us to trigger our approved Chandra Cycle 11 program in 2010 December. We again observed SN 2010j1 in 2011 October and then 2012 June under Cycle 13 of Chandra. The observations were taken with ACIS-S without grating in a VFAINT mode and the duration of the observations at each epoch was $\sim 40 \mathrm{ks}$. In all cases we detected the SN with absorbed count rates of $1.13 \times 10^{-2} \mathrm{cts}$ $\mathrm{s}^{-1}, 3.29 \times 10^{-2} \mathrm{cts} \mathrm{s}^{-1}$ and $3.68 \times 10^{-2} \mathrm{cts} \mathrm{s}^{-1}$, respectively (see Chandra et al. $2012 \mathrm{a}$ for first two epochs).

\section{Results and Interpretation}

\subsection{Supernova 2006jd}

SN 2006jd remained bright in all the radio bands over the four-year span of observations, except for two early upper limits in L band and occasional upper limits in the $\mathrm{K}$ band at later epochs. The radio observations of SN 2006jd show an evolution from a somewhat positive spectral index to a negative index. This type of evolution is commonly observed in radio SNe (Weiler et al. 2002) and is attributed to the transition from optically thick to optically thin radiation. However, the external free-free and synchrotron absorption models are not able to fit the radio data well, which appear to give more of a powerlaw rise. This is also observed in other SNe IIn where the rise of the radio flux was followed: SN 1986J (Weiler et al. 1990) and SN 1988Z (van Dyk et al. 1993, Williams et al. 2002). Weiler et al. (1990) proposed an internal free-free absorption (FFA) model in which thermal absorbing gas is mixed into the synchrotron emitting gas, so that the flux takes the form

$$
\begin{aligned}
F(\nu, t) & =K_{1}\left(\frac{\nu}{5 \mathrm{GHz}}\right)^{\alpha}\left(\frac{t}{1000 \text { days }}\right)^{\beta}\left(\frac{1-\exp \left(-\tau_{\text {intFFA }}\right)}{\tau_{\text {intFFA }}}\right), \\
\tau_{\text {intFFA }} & =K_{3}\left(\frac{\nu}{5 \mathrm{GHz}}\right)^{-2.1}\left(\frac{t}{1000 \text { days }}\right)^{\delta^{\prime}},
\end{aligned}
$$

where $K_{1}$ and $K_{3}$ are the flux and absorption normalizations, respectively. Here $\alpha$ is the optically thin frequency spectral index, which relates to the electron energy index $p$ $\left(N(E) \propto E^{-p}\right)$ as $p=1-2 \alpha$. The assumption that the energy density in the particles and the fields is proportional to the postshock energy density leads to $\beta=3 m-(3-\alpha)(m s+$ $2-2 m) / 2$. Here $m$ is the expansion parameter $\left(R \propto t^{m}, R\right.$ is the shock radius) and $s$ is the CSM density power-law index $\left(\rho \propto r^{-s}\right)$. This model seems to fit the data very well for $s=1.6$ (Fig. 1). The above equation reduces to $F(\nu) \propto \nu^{2.1+\alpha}$ in optically thick limit. The value of $\alpha$ is determined by the late optically thin evolution and is $\alpha=-1.04$ in our case. The fact that the optically thick spectrum is approximately reproduced (Fig. 1) 




Figure 1. Radio light curves of SN 2006jd. Black solid line is the internal FFA model, whereas, the dashed line is the external FFA model.

provides support for the internal FFA model. We find that external absorption played a negligible role in the best-fit case. We also estimate that the mass of the cool gas required to do internal FFA is $M_{a} \approx 2 \times 10^{-8} T_{4}^{5 / 2} M_{\odot}$, showing that a modest amount of cool gas mixed into the emitting region can give rise to the needed absorption (Chandra et al. $2012 \mathrm{~b}$ ). The source of the cool gas is likely to radiative cooling of dense gas in the shocked region.

The XMM-Newton and Chandra X-ray spectra can be fit by either an optically thin thermal spectrum, which leads to an electron temperature $T_{e} \geqslant 20 \mathrm{keV}$, or a nonthermal spectrum with photon index $\Gamma \approx 1.2$. In the nonthermal interpretation, flux $\propto \nu^{0.2}$ (as $\alpha=1-\Gamma$ ) would be a surprisingly hard spectrum for either synchrotron or inverse Compton emission. Thus, we discard the nonthermal origin of X-rays. The X-ray data were best fit with a thermal plasma model at a temperature above $10 \mathrm{keV}$, i.e., in a range where the $0.2-10 \mathrm{keV}$ spectral shape is not sensitive to temperature. The spectrum also indicates a column density of $1.3 \times 10^{21} \mathrm{~cm}^{-2}$, which is significantly larger than the Galactic absorption column density of $4.5 \times 10^{20} \mathrm{~cm}^{-2}$ in that direction. We have a clear detection of $6.9 \mathrm{keV}$ Fe line, which is due to Fe xxvi. A Mekal model with five times the solar abundance does fit the spectrum well and reproduces the iron line at the correct energy but with a narrower width. To look for the possibility of nonequilibrium ionization (NEI), we also fit the XSPEC NEI model to our data. The fit is quite good and it reproduces the iron line at the correct energy. However, the best-fit ionization timescale in the NEI model is $\tau=6.28 \times 10^{11} \mathrm{~s} \mathrm{~cm}^{-3}$. Since $\tau=n t$ ( $n$ is the number density and $t$ is the age) and the XMM spectrum was taken when the SN age was 908 days (or $7.84 \times 10^{7} \mathrm{~s}$ ), this implies a number density of $n=7.7 \times 10^{3} \mathrm{~cm}^{-3}$. This seems low and makes NEI model unlikely.

The Chandra spectrum is best fit with a high-temperature thermal plasma and a column density of $1.6 \times 10^{21} \mathrm{~cm}^{-2}$, which agree with our fits to the XMM-Newton spectrum very well. Even though the Chandra spectrum is not as detailed as that of the XMMNewton, we clearly detect the $6.9 \mathrm{keV}$ Fe line. For Swift observations, we converted the 


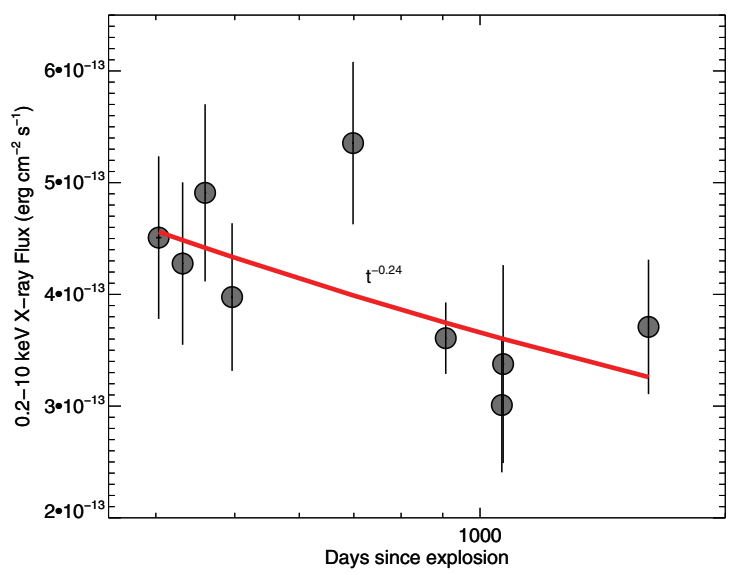

Figure 2. X-ray $0.3-10 \mathrm{keV}$ light curve of SN 2006jd.

count rates to $0.2-10 \mathrm{keV}$ fluxes using a thermal plasma model with a temperature of $60 \mathrm{keV}$ and column density of $1.3 \times 10^{21} \mathrm{~cm}^{-2}$. This gives us X-ray light curve spanning 4 years (Fig. 2). The light curve luminosity $L_{\nu}$ decays as $L_{\nu} \propto t^{-0.24}$. For bremsstrahlung emission $L_{\nu} \propto t^{2 m(1-s)+1}$. This implies $s=1.7$ for $m=0.9$. The radio and X-ray observations thus give $s \approx 1.6$.

In the thermal interpretation, the temperature $T$ is assumed to be produced by shock heating, with the shock velocity $v_{s h}=\left[16 k T /\left(3 \mu m_{p}\right)\right]^{1 / 2}=6700(k T / 60 \mathrm{keV})^{1 / 2} \mathrm{~km} \mathrm{~s}^{-1}$, where $k$ is Boltzmann's constant, $\mu$ is the mean molecular weight, and $m_{p}$ is the proton mass. The shock velocity is closer to expectations for the forward shock than the reverse shock wave at this age, although X-ray emission from young SNe is typically attributed to the reverse shock wave (Chevalier \& Fransson 2003). Using results from Fransson et al. (1996) for $L_{\nu}$, the mass-loss rate $\dot{M}$, normalized to $R=10^{15} \mathrm{~cm}$, is $\dot{M}_{-3} / v_{w 2} \approx 5 v_{4}^{0.6}$, where $\dot{M}_{-3}=\dot{M} /\left(10^{-3} M_{\odot} \mathrm{yr}^{-1}\right), v_{w 2}$ is the preshock wind velocity in units of $100 \mathrm{~km}$ $\mathrm{s}^{-1}$, and $v_{4}$ is the average velocity in units of $10^{4} \mathrm{~km} \mathrm{~s}^{-1}$ at $10^{3}$ days. At $10^{3}$ days, the preshock density is $2 \times 10^{18} v_{4}^{-1} \mathrm{~g} \mathrm{~cm}^{-3}$. The corresponding density in the shocked gas is $n_{H} \sim 3 \times 10^{6} \mathrm{~cm}^{-3}$.

A robust result of fitting the X-ray spectrum is the hydrogen column density $N_{H}$ to the source. Of the $N_{H}=1.3 \times 10^{21} \mathrm{~cm}^{-2}$ that is observed, $4.5^{20} \mathrm{~cm}^{-2}$ can be attributed to the Galaxy, so there is $8.5 \times 10^{20} \mathrm{~cm}^{-2}$ left for the SN host galaxy interstellar medium and the SN CSM. This column density estimate assumes that the absorbing medium has solar abundances, and is cool and not highly ionized. In the model for the X-ray emission discussed above, the expected column density in the unshocked CSM is $410^{22}$ $\mathrm{cm}^{-2}$, a factor of 50 larger than that inferred from the observations. A possible reason is that the CSM is fully ionized by the X-ray radiation. However, the ionization parameter for this case is $\xi=L / n r^{2}=50$, which indicates that there may be some ionization of CNO elements, but not full ionization of the gas Verner \& Ferland 1996. To check on this result, we ran the CLOUDY code Ferland et al. 1998 for the parameters discussed above. As expected, we found some ionization of CNO elements, but little change in the absorption of the X-ray spectrum. The radio data also suggests that the FFA along the line of sight to the X-ray emission fails to produce the observed radio absorption. This is consistent with our analysis of the radio emission, which showed that external FFA does not lead to good model fits to the data. 


\subsection{Supernova 2010jl}

In Fig. 3, we plot the spectra of Dec 2010 and Oct 2011 Chandra observations. The fluorescent $6.4 \mathrm{keV} F$ line is present in the first spectrum but not the second. The lower limits on the temperature for the two spectra are $8 \mathrm{keV}$ and $12 \mathrm{keV}$, respectively, showing that a hot component is present. The column densities of the main X-ray emission component are high with values of $\sim 10^{24} \mathrm{~cm}^{-2}$ and $3 \times 10^{23} \mathrm{~cm}^{-2}$ (for a metallicity of $\left.Z \sim 0.3 Z_{\odot}\right)$, respectively. These are 3000 times and 1000 times higher than the Galactic column density $\left(3 \times 10^{20} \mathrm{~cm}^{-2}\right)$. The high value and variability of $N_{H}$ point to an origin in the CSM. The excess column density to the X-ray emission is not accompanied by high extinction to the SN, showing that the column is probably due to mass loss near the forward shock wave where any dust has been evaporated. This is the first time that external circumstellar X-ray absorption has been clearly observed in an SN. The unabsorbed emission from the SN is constant within $20 \%-30 \%$ at the two epochs, with $\sim 7 \times 10^{41} \mathrm{erg} \mathrm{s}^{-1}$, placing SN 2010jl among the most luminous X-ray SNe yet observed. In the thermal interpretation, a lower limit of $10 \mathrm{keV}$ for the temperature puts a lower limit of the $2700 \mathrm{~km} \mathrm{~s}^{-1}$ on the shock speed. In comparing the observed luminosity to a thermal emission model to find the physical parameters, we note that our measurements give the spectral luminosity, not the total luminosity. We use Equation (3.11) of Fransson et al. (1996) for the luminosity. These expressions allow for a variation of the pre-shock density $\propto r^{-s}$, where $s$ is a constant. For the plausible value $m=0.8$, we find that $s=1.6$ gives a reasonable representation of the luminosity and $N_{H}$ evolution. The implied value of the mass loss rate $\dot{M}$, normalized to $R=10^{15} \mathrm{~cm}$, is $\dot{M}_{-3} / v_{w 2} \approx 8 v_{4}^{0.6}$, where $\dot{M}_{-3}=\dot{M} /\left(10^{-3} M_{\odot} \mathrm{yr}^{-1}\right), v_{w} 2$ is the pre-shock wind velocity in units of $100 \mathrm{~km}$ $\mathrm{s}^{-1}$, and $v_{4}$ is the shock velocity in units of $10^{4} \mathrm{~km} \mathrm{~s}^{-1}$ at the first epoch. The high temperature implies that we are observing the forward shock region. In modeling the Xray absorption in SN 2010jl we have assumed that the absorbing gas is not fully ionized. If the circumstellar gas is photoionized by the X-ray emission, the absorption is reduced (e.g., Fransson 1982).

The 2010 December spectrum shows a $6.4 \mathrm{keV}$ feature, which is identified with the narrow $\mathrm{K} \alpha$ iron line. Since the $6.4 \mathrm{keV}$ Fe line arises from neutral or low ionized iron (Fe I to Fe XI), it supports our finding that the radiation field is not able to completely ionize the circumstellar gas. At the second epoch, $N_{H}$ is smaller by a factor of three, so the strength of the Fe line should be correspondingly smaller; this is consistent with the nondetection of the line. The problem with this picture is that it assumes $\mathrm{Fe}$ is in the low ionization stages that produce the $\mathrm{K} \alpha$ line; this requires an ionization parameter $\xi \leqslant 5$ (Kallman et al. 2004), which is below the inferred value. One possibility is that the circumstellar gas is clumped, with a density $\geqslant 40$ times the average; another is that the $\mathrm{K} \alpha$ line emission is from dense gas that is not along the line of sight.

A thermal fit to the low-temperature component (Fig. 3) implies an absorbing column density of $(1.37 \pm 8.44) 10^{20} \mathrm{~cm}^{-2}$, much less than the column to the hot component and consistent with the Galactic column density within the errors. This rules out the possibility that the cooler X-rays come from slow cloud shocks in the clumpy CSM or from the reverse shocks. The component is also present in the second epoch. It could arise from a pre-SN mass loss event or from an unrelated source in the direction of the SN. The components are best fit with either a thermal component $(T \sim 1-2 \mathrm{keV})$ or a power law with $\Gamma=1.6-1.7$. The luminosities of this component in the 2010 December and 2011 October spectra are $3.5 \times 10^{39} \mathrm{erg} \mathrm{s}^{-1}$ and $5 \times 10^{39} \mathrm{erg} \mathrm{s}^{-1}$, respectively. The luminosity range and the power-law index are compatible with a background ultraluminous X-ray source (ULX), which can typically be described by an absorbed power-law spectrum 

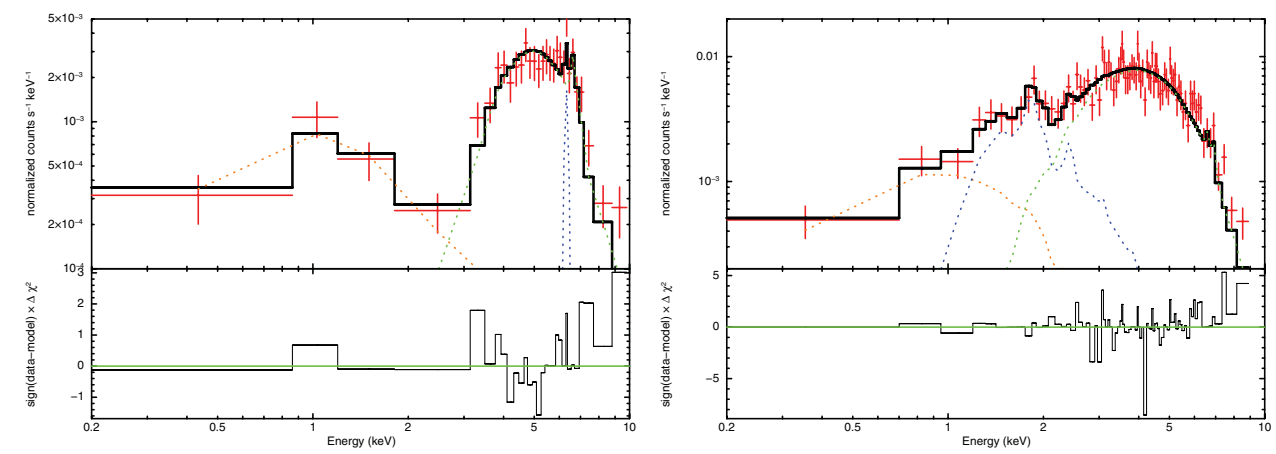

Figure 3. Chandra spectra of SN 2010jl taken in Dec 2010 (left) and in Oct 2011 (right). The presence of $\mathrm{Fe} 6.4 \mathrm{keV}$ line in the first spectrum is evident. There is also an indicating of an extra component (around $1 \mathrm{keV}$ ) in the Oct 2011 spectrum.

Swartz et al. 2004. Since the error in the flux determination is between $20 \%-30 \%$, a factor of 1.4 change in the luminosity at the two epochs is consistent with a constant flux. Thus we attribute this component to a background source, most likely a ULX, which is associated with the blue excess emission region seen in the pre-SN HST images Smith et al. 2011. There is an additional low temperature component present in the second epoch which was not present in the Dec 2010 spectrum. Origin of this component is not clear.

\section{Discussion and Conclusions}

The basic interpretation of the X-ray and radio data in terms of standard spherical models led to inconsistencies in SN 2006jd. One is that the column density of matter to the X-ray emission is about a factor 50 smaller than that needed to produce the X-ray luminosity. The straightforward explanation is that there is a global asymmetry in the distribution of the circumstellar gas that allows a low column density in one direction, while dense interaction is taking place over much of the rest of the solid angle (as viewed from the SN). Chugai \& Danziger (1994) had suggested a scenario with equatorial mass loss to explain the presence of fast- and intermediate-velocity shock fronts in the Type IIn SN 1988Z. Polarization observations of SNe IIn have shown evidence for large-scale asymmetry, e.g., SN 1997eg Hoffman et al. 2008, SN 1998S Leonard et al. 2000, and SN 2010jl Patat et al. 2011. In this view, the column density to the radio emission would be small, as for the X-ray emission, because they are both from the same region. The external FFA model for the radio absorption thus fails, and an absorption mechanism internal to the emission is indicated. We have argued that thermal absorption internal to the emitting region is a plausible mechanism, as has previously been proposed for other SNe IIn Weiler et al. 1990. There is evidence that the X-ray properties we have found for SN 2006jd are not unusual for a Type IIn event. Chandra observations of SN 2001em and SN 2005dk have shown hard emission Pooley \& Lewin 2004, Pooley et al. 2007, implying a high temperature. Our observations at both radio and X-ray wavelengths imply that the circumstellar density profile $\left(\rho \propto r^{-2}\right)$ is flatter than the $s=2$ case that would be expected for a steady wind. We find $s=(1.5-1.6)$; deviation from the steady case is plausible for a SN IIn because the mass loss may be due to an eruptive event. In addition, the optical luminosity evolution of SN 2006jd shows a second peak at $\sim 500$ days Stritzinger et al. 2012 that is indicative of a nonstandard density distribution. 
In SN 2010j1 2010 December spectrum has only one temperature component associated with the high column density. However, in the 2011 October spectrum, there are two temperature components associated with a high column density, one with temperature $\sim 10$ $\mathrm{keV}$ and another with temperature $1.1 \mathrm{keV}$. The lower temperature component fits with $1 / 4$ the column density of the high-temperature component. The fact that the component is absent at the first epoch suggests that it is related to the SN emission. We examined the possibility that the emission is the result of reduced absorption due to photoionization of the absorbing material, in particular, that lighter atoms are ionized but heavier atoms are not. However, we were not able to reproduce the observed emission and the source of this emission remains uncertain. SN 2010jl is a special Type IIn SN because we have been able to catch it in X-rays early on with as sensitive an instrument as Chandra and trace the early X-ray evolution. We observe dramatic changes over two epochs separated by 10 months. For the first time we see clear evidence of external CSM absorption in an $\mathrm{SN}$. We also find that the CSM is not fully photoionized by the SN emission, the SN is very luminous in X-rays, and the temperature of the emitting gas is $\geqslant 10 \mathrm{keV}$.

\section{References}

Benetti, S., Bufano, F., Vinko, J., et al. 2010, CBET, 2536, 1

Blondin, S., Modjaz, M., Kirshner, R., et al. 2006, CBET, 679, 1

Chandra, P., Chevalier, R. A., Irwin, C. M., et al. 2012, ApJ, 750, L2

Chandra, P., Chevalier, R. A., Chugai, N., et al. 2012, ApJ, 755, 110

Chandra, P. \& Soderberg, A. 2007, ATel, 1297, 1.

Chevalier, R. A. \& Fransson, C. 2003, in: K. Weiler (eds.), Supernovae and Gamma-Ray Bursters: Lecture Notes in Physics (Berlin: Springer), vol. 598, p. 171

Chevalier, R. A. 1982, ApJ, 259, 302

Chugai, N. N. \& Danziger, I. J. 1994, MNRAS, 268, 173

Ferland, G. J., Korista, K. T., Verner, D. A., et al. 1998, PASP, 110, 761

Filippenko, A. V. 1997, ARAA, 35, 309

Fransson, C., Lundqvist, P., \& Chevalier, R. A. 1996, ApJ, 461, 993

Fransson, C. 1982, A\&A, 111. 140

Hoffman, J. L., Leonard, D. C., Chornock, R., et al. 2008, ApJ, 688, 1186

Immler, S., Milne, P., \& Pooley, D. 2010, ATel, 3012, 1

Immler, S., Brown, P. J., Filippenko, A. V., \& Pooley, D. 2007, ATel, 1290, 1

Kallman, T. R., Palmeri, P., Bautista, M. A., Mendoza, C., \& Krolik, J. H. 2004, ApJS, 155, 675

Kiewe, M., Gal-Yam, A., Arcavi, I., et al. 2012, ApJ, 744, 10

Leonard, D. C., Filippenko, A. V., Barth, A. J., \& Matheson, T. 2000, ApJ, 536, 239

Newton, J. \& Puckett, T. 2010, CBET, 2532, 1

Patat, F., Taubenberger, S., Benetti, S., Pastorello, A., \& Harutyunyan, A. 2011, A\&A, 527, L6

Pooley, D., Immler, S., \& Filippenko, A. V. 2007, ATel, 1023, 1

Pooley, D. \& Lewin, W. H. G. 2004, IAU Circ., 8323, 2

Prasad, R. R. \& Li, W. 2006, CBET, 673, 1

Schlegel, E. M. 1990, MNRAS, 244, 269

Smith, N., Li, W., Miller, A. A., et al. 2011, ApJ, 732, 63

Stoll, R., Prieto, J. L., Stanek, K. Z., et al. 2011, ApJ, 730, 34

Stritzinger, M., Taddia, F., Fransson, C., et al. 2012, ApJ 756, 173

Swartz, D. A., Ghosh, K. K., Tennant, A. F., \& Wu, K. 2004, ApJS, 154, 519

van Dyk, S. D., Weiler, K. W., Sramek, R. A., \& Panagia, N. 1993, ApJ, 419, L69

Verner, D. A. \& Ferland, G. J. 1996, ApJS, 103, 467

Weiler, K. W., Panagia, N., Montes, M. J., \& Sramek, R. A. 2002, ARAA, 40, 387

Weiler, K. W., Panagia, N., \& Sramek, R. A. 1990, ApJ, 364, 611

Williams, C. L., Panagia, N., Van Dyk, S. D., et al. 2002, ApJ, 581, 396 


\section{Discussion}

PODSIADLOWSKI: Two questions: 1. Your modeling of the CSM assumes spherical symmetry. In many models for the mass ejection (and there is probably also observational evidence for this), the medium is very anisotropic. Is there a way to model the radio emission for that case? 2. In your modeling of SN 2006jd you said that the Metal fits required a metallicity of 5 times solar, which seems very extreme. Is there another way to explain the data?

CHANDRA: 1: In radio as of now we do assume spherical symmetry to model the radio emission. It may not be a bad assumption because in VLBI of some supernovae, such as SN 1993J, the emission was quite spherical. One can model the circumstellar inhomogeneities but I am not aware of any radio emission model which takes care of the global asymmetry in the explosion. However, in Type IIn supernovae, circumstellar density clumps can be models in radio emission.

2: We did try various models including non-equilibrium ionization models. The only way the energy and width of the strong iron line would fit would be by assuming 5 times solar metallicity.

CHAKRABORTI: Is the gap between the two observations larger than the cooling time of the forward shocked gas? Why does the Fe line vanish?

CHANDRA: 1. SN 2006jd was classified as Type IIn supernova only after 2 years post discovery. And thats when we started following it in the radio bands regularly. The early post discovery observations were taken only once where it was not detected. So we obviously missed the cooling timescale window. 2. The Iron line vanishes in SN 2010j1 two spectra because the column density is 3 times smaller in the second spectrum, which means strength of Iron line should be 3 times smaller than the previous detection, which is consistent with the non-detection.

POOLEY: Concerning the ULX component of the spectrum, why does it fall off so quickly? (Note: I thought you said it had a power-law index of 1.7)

CHANDRA: ULX components usually have power law of this order. Our results are indeed consistent with the ULX interpretation.

WANG: One needs to treat the parameters from the fits of the assumed model with grain of salt, since the model could not really be tested. For example, a combination of a thermal + Power law would give quite different parameters.

CHANDRA: Yes, the combination would have given different fit but would have increased number of free parameters. Yes, fits should be taken with grain of salt but we are trying to fit with a simplistic model which can best represent the data. 